\title{
Monopole annihilation in cosmic necklaces
}

\author{
Jose J. Blanco-Pilladd* and Ken D. Olum \\ Institute of Cosmology, Department of Physics and Astronomy, \\ Tufts University, Medford, MA 02155
}

\begin{abstract}
A sequence of two symmetry breaking transitions in the early universe may produce monopoles whose flux is confined into two strings each, which thus assemble into "necklaces" with monopoles as beads. Such "cosmic necklaces" have been proposed as a source of ultra-high-energy cosmic rays. We analyze the evolution of these systems and show that essentially all monopoles annihilate or leave the string at early times, after which cosmic necklaces evolve in a similar way to a network of ordinary cosmic strings. We investigate several modifications to the basic picture, but in nearly all cases we find that too few monopoles remain on the necklaces to produce any observable cosmic rays. There may be a small window for superconducting condensates to prevent annihilations, but only if both the string and the condensate scale are very high.
\end{abstract}

PACS numbers: $98.80 . \mathrm{Cq} 98.70 . \mathrm{Sa}$

*Electronic address: jose@cosmos.phy.tufts.edu.edu

$\dagger$ Electronic address: kdo@cosmos.phy.tufts.edu 


\section{INTRODUCTION}

Conventional acceleration mechanisms within known astrophysical objects face serious difficulties in accounting for the observation of the ultra-high energy cosmic rays (UHECR's) with energies above $10^{20} \mathrm{eV}$ [1]. This has motivated the search for alternative scenarios that could explain the origin of such stupendous energies.

Topological defects (for a review see [2, 3]) are in this regard a very natural candidate to produce high energy radiation that could explain the UHECR observations. On the one hand, they are created during a phase transition in the early universe and are therefore associated with an energy scale typically much higher than $10^{20} \mathrm{eV}$. On the other hand, due to their topological stability they can survive for long periods of time, allowing them to release this energy today, when the universe is much colder and their energies are very atypical. Several such scenarios have been proposed in the literature based on different kinds of defects such as monopole-antimonopole pairs, cosmic strings, superconducting strings or hybrid defects. (See [4, 5] and references therein).

In this paper we will consider in detail the model proposed in [6] based on the hybrid defects known as "cosmic necklaces". This type of defects can be produced due to a sequence of symmetry breaking phase transitions with either of the following structures,

$$
\begin{aligned}
& G \rightarrow U(1) \rightarrow Z_{2}, \\
& G \rightarrow H \times U(1) \rightarrow H \times Z_{2},
\end{aligned}
$$

where $G$ is a semi-simple group. In both of these scenarios, the first transition produces monopoles of mass $M \sim \eta_{M} / e$, where $\eta_{M}$ characterizes the energy scale of the universe at that time. The second transition at a lower scale $\eta_{s}$ gives rise to cosmic strings of energy per unit length given by ${ }^{1} \mu \sim \eta_{s}^{2}$.

Each string confines half of the $U(1)$ magnetic flux coming from a monopole. ${ }^{2}$ This leads to a situation in which each monopole is attached to two strings, each of which has an antimonopole on the other end. This process creates a network of infinite strings and loops with monopoles spaced along the strings ${ }^{3}$, similarly to beads on a necklace, giving rise to the name "cosmic necklaces". Cosmic necklaces have been considered an attractive source for ultra-high-energy cosmic rays [6], because the monopoles could annihilate at late times to produce high energy particles. ${ }^{4}$

Particle physics models with this type of symmetry breaking pattern have been previously discussed in the literature [9, 10]. Similar objects, although with somewhat different properties, have also been discussed more recently within the string theory context [11-16].

The cosmological evolution of monopoles depends on the distribution of energy between the monopoles and the strings. The essential parameter [6] is the ratio of the energy in

\footnotetext{
${ }^{1}$ We use units in which $c=\hbar=k_{B}=1$ throughout.

2 Thus this $U(1)$ cannot be the usual electromagnetism, but some different field whose flux is confined. In the case of Eq. (1b), the monopole may also be the source of other fluxes that are not confined.

3 We assume here that the monopoles and antimonopoles are randomly located. If instead the particles are in nearby pairs, for example as in Ref. 7], the two strings from a given monopole would often go to the same antimonopole, leading to two-particle loops similar to the strings discussed in Ref. [8], with few long necklaces.

${ }^{4}$ In the case of Eq. (1a), we imagine that there is some very-high-energy connection between the group G and the standard model, which permits monopoles to decay into ordinary particles.
} 
monopoles to that in string segments,

$$
r=\frac{M}{\mu d}
$$

where $d$ is the average distance between monopoles. If $r \ll 1$, the string evolves essentially without regard to the monopoles. The monopoles in such a necklace move along the string like beads on a moving wire without significant back reaction on the motion of the string itself.

On the other hand, if $r$ is significant the motion is different from an ordinary cosmic string without monopoles. The typical velocity is [6]

$$
v \sim 1 / \sqrt{1+r}
$$

If $r \gg 1$, then $v \sim 1 / \sqrt{r}$, which one can verify by treating the necklace as a nonrelativistic string with tension $\mu$ but mass per unit length $r \mu$.

Ref. [6] studied the evolution of the parameter $r$ neglecting the effect of monopole annihilation and concluded that the system may evolve towards a state with $r \gg 1$. On the other hand, Ref. [17] studied the dynamical evolution of loops with $r \ll 1$ and found that in this case monopole annihilation is very efficient. This is not very surprising, taking into account that in this limit, the monopoles have no significant effect on the system and they are therefore thrown around by the motion of the string. This process gives a longitudinal velocity to the monopoles on the strings close to the characteristic transverse string velocity, which in this case is relativistic [17].

The parameter space of models described in [6] is very wide due to the vast variation of possible scales allowed for the monopole mass $M$ (whose only restriction is to be above the highest energy observed in the UHECR spectrum, $M>3 \times 10^{11} \mathrm{GeV}$ ) as well as the string tension $\mu$. On the other hand, it is clear that the necklace network should be able to release enough energy to explain the UHECR and therefore we should ensure that the fraction of the critical density in the monopoles on the necklaces is larger than that in UHECR,

$$
\Omega_{M}>\Omega_{\mathrm{UHECR}} .
$$

For a scaling distribution of cosmic strings moving relativistically, we expect $\Omega \sim G \mu=$ $\mu / m_{\mathrm{pl}}^{2}$ [2]. For a network moving with speed $v$, all scales on the string network will be larger at a given time by a factor $1 / v[6]$, so the contribution from strings is $\Omega \sim G \mu / v^{2}$. The mass in monopoles is $r$ times that in strings, so

$$
\Omega_{M} \sim \frac{r(1+r) \mu}{m_{\mathrm{pl}}^{2}} .
$$

To match the observed flux of UHECR with the model of Ref. [6] implies $\Omega_{\mathrm{UHECR}} \sim 10^{-10}$, so we require

$$
\Omega_{M} \gtrsim 10^{-10}
$$

for a viable model.

In this paper, we show that monopole annihilation in cosmic necklaces is in fact a very efficient process to the point that we have not been able to find any region of the parameter space in simple models that satisfies the above requirement. Monopole-antimonopole 
interactions rapidly annihilate monopoles or break off monopole-antimonopole pairs. The remaining string behaves as a normal cosmic string network while the pairs evolve as described in [8].

The plan of this paper is as follows. We first consider the simple case of smooth, nonsuperconducting, Type II (or BPS) strings. In section II we show that monopoles acquire a typical velocity along the strings similar to the transverse string velocity $v$. In section III, we investigate what happens when a monopole and an antimonopole come together along the string. We discuss the probabilities for annihilation, reflection, and expulsion of the pair from the string. In section IV we calculate the effects of these processes and show that the monopole density rapidly decreases.

In section $\nabla$ we study the effect of correlations and statistical fluctuations in monopole velocities on the conclusions of this paper. In section VI, we consider how wiggliness affects the monopole motion. InVII, we consider the case of monopoles which have some fluxes that are not confined, in addition to the flux that forms the strings. In section VIII we consider type I strings, which attract each other and so make the detachment process less common. In all these cases, we find our conclusions about rapid annihilation unchanged. In section IX. we consider the case of strings with superconducting condensates, which can oppose the motion of the monopoles along the string. In this case we find that if the condensate particles are trapped between monopoles, there is a small window of parameter space in which annihilations are prevented and so there may be the possibility of a viable model. We conclude in section $\mathrm{X}$.

\section{MONOPOLE MOTION}

If the two strings attached to a monopole point in opposite directions, there is no net force on the monopole. The monopole can move freely along the string, reducing the length of the string on one side and increasing the length on string on the other. In the limit $r \ll 1$, the back reaction of the monopole on the string is small, and we can treat the monopoles as beads sliding freely along a moving wire.

How rapidly should the typical monopole move along the string? In certain very simple cases, such as a string with standing waves and monopoles at the nodes or antinodes, the monopoles will not move. But in general, the motion of the string can give longitudinal velocities to the monopoles.

As a simple example, consider a $r \ll 1$ necklace where the string is made up of a series of kinks and the motions of the different segments are uncorrelated. Suppose a monopole has longitudinal velocity $v_{\|}$along a string segment moving with velocity $v$. Since the monopole is constrained to move with the string, its transverse velocity is also $v$, and its overall velocity is given by $v_{M}^{2}=v^{2}+v_{\|}^{2}$.

Now suppose the monopole suddenly finds itself on a different string segment making angle $\theta$ with the overall direction of monopole motion. The monopole now has a new longitudinal velocity $v_{\|}^{\prime}=v_{M} \cos \theta$ so $v_{\|}^{\prime 2}=\cos ^{2} \theta\left(v^{2}+v_{\|}^{2}\right)$.

Let us average over all realizations and look for a stationary mean squared monopole velocity. We find $\left\langle v_{\|}^{\prime 2}\right\rangle=\left\langle\cos ^{2} \theta\right\rangle\left(\left\langle v^{2}\right\rangle+\left\langle v_{\|}^{2}\right\rangle\right)$. By assumption the old and new string directions are uncorrelated, so $\left\langle\cos ^{2} \theta\right\rangle=1 / 3$, and $\left\langle v_{\|}^{\prime 2}\right\rangle=\left\langle v^{2}\right\rangle / 2$. We will not be concerned with the factor of 2 , only with the fact that the longitudinal monopole velocity is comparable to the string velocity. 
A realistic string would be much more complicated than the simple model above. There would be not only sharp kinks but also smooth variations of direction and velocity. It would also be possible for energy to flow back and forth between the string and the monopole. In this case, we can make an argument of equipartition. The average energy in the string between monopoles is $\mu d$. The energy of a monopole with velocity $v_{M}$ is $M v_{M}^{2} / 2$ in the nonrelativistic case. For these to be roughly equal, we should have $v_{M}$ of order $\sqrt{\mu d / M}=$ $1 / \sqrt{r}$. The monopole kinetic energy should be divided between longitudinal and transverse motion, both of which should then have velocities of order $1 / \sqrt{r}$. Thus once again we conclude that the longitudinal monopole velocity is of order $1 / \sqrt{r}$ if $r \gg 1$.

The motions of adjacent monopoles are not independent. The string will be smooth on some correlation length $\xi$. Monopoles within distance $\xi$ will be subjected to similar motions and will thus tend to acquire similar longitudinal velocities. As a simple model, we can take monopoles to consist of trains of order $\xi / d$ monopoles all moving similarly with random longitudinal velocities of order $v$.

\section{MONOPOLE INTERACTION}

As discussed in the previous section, the monopoles will acquire velocities along the string. Suppose a monopole and an antimonopole are moving toward each other. Unless some process causes them to turn around again (which will be the subject of later sections), the monopole and antimonopole will come together. What will happen then?

First suppose that the string is straight and the monopole velocity is entirely along the string. If the monopole and antimonopole collide head-on, they will annihilate. The monopole mass-energy will then be radiated in high-energy particles and lost to the necklace.

However, if the string scale and the monopole scale are well separated, the monopoles might easily pass by each other inside the string, as shown in Fig 1(a)-(b). There is now an string with winding number $n=3$ between the monopoles and $n=1$ strings outside them. They thus feel a net force pulling them back toward each other. In the critically coupled (BPS) case, the force is $2 \mu$. For Type II strings it is even larger. The Type I case will be discussed in Sec. VIII,

In an ideal geometry, this force would eventually reverse the monopole velocities so that they pass back past each other. However, during the time that the monopoles have passed, there is no longer any topological constraint holding them to the string, and it is possible for them to detach as shown in Fig 1(c).

If the string is Type II, a string with winding number $n=2$ and another with $n=1$ have less energy than a single $n=3$ string. It is therefore favored for the monopole pair to detach itself from the rest of the string, forming an $n=2$ string (or two $n=1$ strings) containing all the flux between monopole and antimonopole. If instead the string has critical coupling, there is no energetic advantage or disadvantage to detachment. If the string is not exactly straight, however, the monopoles have some transverse velocity which will encourage detachment. If the strings are Type I, detachment is energetically disfavored. Such strings will be the subject of section VIII.

If the monopoles do not detach themselves from the string, the extra tension of the $n=3$ string will eventually pull them back past each other. This gives another chance at annihilation, but if that fails the net effect will be that the monopole and antimonopole bounce and recede from each other. 

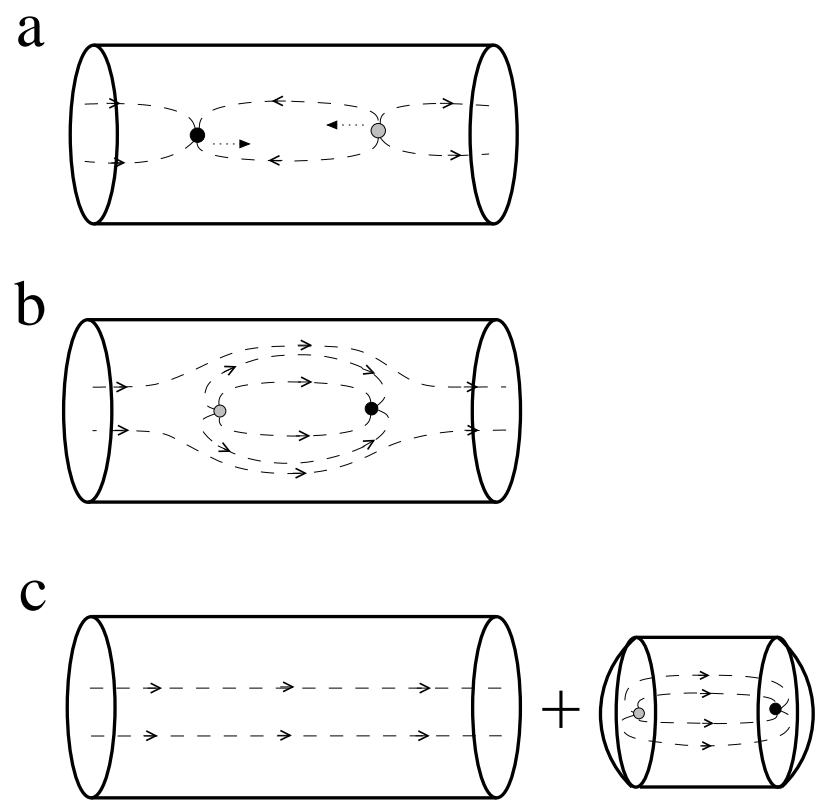

FIG. 1: Monopole (gray) and antimonopole (black) passing by inside a string. Dashed lines with arrows show the magnetic flux lines. First (a) the particles are moving towards each other inside the string. After they pass there are 3 strings worth of flux in the region between the monopoles. By rearranging flux lines (b), all flux from the monopole goes to the antimonopole, so the monopoleantimonopole pair can detach itself from the rest of the string (c).

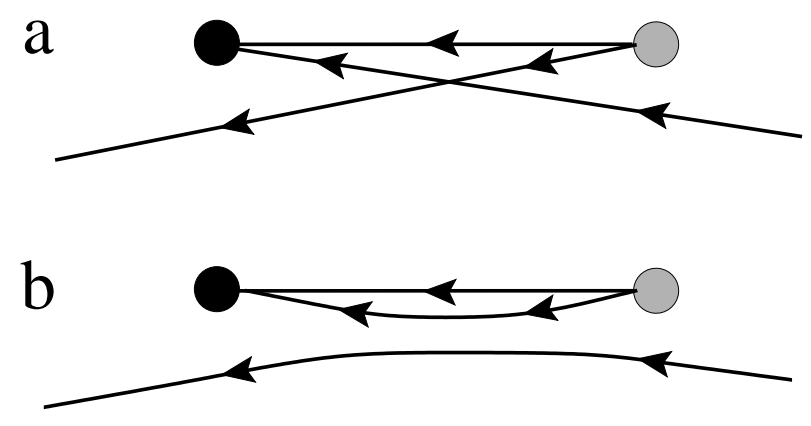

FIG. 2: A monopole and an antimonopole can pass each other to form a zigzag string (a). Some of the time there will then be a reconnection (b) which allows the monopole-antimonopole pair to detach itself from the rest of the string.

If the monopole velocities are significantly misaligned, the minimum distance of approach will be larger than the string radius. In that case the monopoles will pass outside the string and the result will be a "zigzag" string, as shown in Fig. 2. There is now a nontrivial chance that the strings will cross and reconnect. Then, as above, the monopoles are detached into a 2-monopole loop, leaving the rest of the string behind. This is somewhat similar to the situation described in [17], where non-periodic oscillations of a loop led to frequent intercommutations. If this does not happen, each monopole feels a force $2 \mu$ pulling it back in the direction from which it came, so eventually monopole and antimonopole velocities will be reversed and again monopoles will have reflected.

We will describe the result of a close approach between monopole and antimonopole with 
a probability $P_{a}$ for annihilation, $P_{d}$ for detachment, and $P_{r}=1-P_{a}-P_{d}$ for reflection. For most purposes, we do not need to distinguish between annihilation and detachment, so we write $P_{x}=P_{a}+P_{d}$ for the probability of any process that removes the monopoles from the string.

\section{RESULTING MONOPOLE DENSITY}

In the previous section we argued that (at least in the simple case of smooth, nonsuperconducting, non-type-I strings), the probability of annihilation or detachment, $P_{x}$, is significant. Let us now estimate how this affects the value of $r$.

At very early times, the network of necklaces is dominated by friction. Necklaces therefore do not move even with velocity $v$ so the monopoles are not significantly annihilated (although, even in this case, $r$ is decreased by the expansion of the universe). As we discuss in the Appendix, this era ends at a time $t_{\text {necklace }}$ at which it becomes possible for the necklaces to move at the characteristic velocity $v \sim 1 / \sqrt{1+r}$. If $r$ is significant, $t_{\text {necklace }}$ is earlier than the usual frictional timescale $t_{*}$ where strings without monopoles can move relativistically, because of the slower characteristic velocity of necklaces.

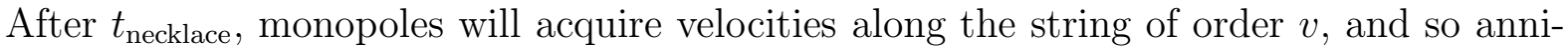
hilate or detach if $P_{x}$ is large. In the absence of friction this process increases the monopole distance to $d \sim v t$. Particles further away than this have had no opportunity to encounter each other. As $r$ declines, $v$ increases, and thus $r$ decreases at least as $t^{-1}$. In the radiation

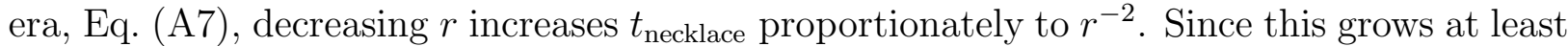
as fast than $t$, friction limits the growth of $v$ and the necklaces stay at the edge of the frictional regime. ${ }^{5}$ In the matter era, Eq. (A8), decreasing $r$ increases $t_{\text {necklace proportionately }}$ to $r^{-1}$, so $t_{\text {necklace }}$ keeps pace with $t$. Thus in either case we stay at the edge of the frictional regime, until we arrive at $t_{*}$ with $r \sim 1$. Except for very light strings, $t_{*}$ is well earlier than the age of the universe today, $t_{0}$. Since $r \lesssim 1$ at $t \gtrsim t_{*}, v$ is of order 1 from $t_{*}$ until $t_{0}$. (See Eq. [3.) Then by $t_{0}$ the distance between monopoles increases to

$$
d \sim t_{0} \sim 10^{41} \mathrm{GeV}^{-1}
$$

and so

$$
r=\frac{M}{\mu d} \sim \frac{M}{\mu t_{0}} \ll 1
$$

This gives

$$
\Omega_{M} \sim \frac{M}{m_{\mathrm{pl}}^{2} t_{0}}<10^{-62}
$$

so the effects of monopoles are completely negligible.

If the string scale is less than about

$$
\mu_{*}=\frac{m_{\mathrm{pl}}^{2}}{t_{0} T_{\mathrm{eq}}} \sim\left(10^{3} \mathrm{GeV}\right)^{2},
$$

\footnotetext{
${ }^{5}$ In fact annihilations take place even when the necklace speed is restricted by friction, but we have ignored those. Taking them into account would make the effect of monopoles even less important.
} 
$t_{*}$ is still in the future. In that case, we can have $r>1$ today. But we are still limited by the constraint that the necklaces should be at the edge of the frictional regime. Then from Eq. (A8),

$$
r \sim \frac{m_{\mathrm{pl}}^{2}}{\mu t_{0} T_{\mathrm{eq}}}
$$

Since $r>1$, Eq. (51) gives

$$
\Omega_{M} \sim \frac{m_{\mathrm{pl}}^{2}}{\mu t_{0}^{2} T_{\mathrm{eq}}^{2}}<10^{-30}
$$

if the lightest possible strings have energy scale $100 \mathrm{GeV}$. This is still 20 orders of magnitude too low to satisfy Eq. (6)

In fact, this conclusion holds even if the probability of annihilation or detachment is quite small. If a monopole-antimonopole interaction does not lead to annihilation or detachment from the string, the particles effectively bounce. After that they head away from each other and have an opportunity to annihilate with their partners on their other sides. Thus in the case where $P_{x}$ is significantly less than 1 , monopoles and antimonopoles move back and forth with velocity $v$ on segments of string of length $d$, and so have opportunities to interact separated by a time $d / v$. The timescale of annihilation or detachment is then $t_{x}=d /\left(v P_{x}\right)$. A value of $r$ that gives $t_{x}$ earlier than the present time would have decreased before now, so the largest $r$ that can currently exist is found by setting $t_{x}=t_{0}$. Using $d=M /(\mu r)$, $v \sim 1 / \sqrt{1+r}$, we find

$$
\frac{r}{\sqrt{1+r}} \sim \frac{M}{\mu t_{0} P_{x}}
$$

Taking extreme values of the parameters to make $r$ as large as possible, namely $M=$ $10^{17} \mathrm{GeV}$ and $\mu=(100 \mathrm{GeV})^{2}$, and $t_{0} \sim 10^{41} \mathrm{GeV}^{-1}$, we find that the right hand side is at most $10^{-28} P_{x}^{-1}$. Thus providing $P_{x}>10^{-28}, r<1$ and

$$
\Omega_{M} \sim \frac{M}{m_{\mathrm{pl}}^{2} t_{0} P_{x}}<10^{-34}
$$

Even smaller values of $P_{x}$ will be discussed in Sec. VIII.

Thus we find that in the simple cases considered so far, monopole annihilation or detachment reduces the number of monopoles on necklaces to negligible values, so that they cannot act as a source of ultra-high-energy cosmic rays. In the sections below, we will discuss several different modifications of this picture.

\section{CORRELATIONS AND FLUCTUATIONS OF MONOPOLE MOTION}

As described in section [II, the velocities of adjacent monopoles will be correlated, so that there are trains with alternating monopoles and antimonopoles all moving with similar velocities. Particles within a train will not approach at velocity $v$, as we assumed before, so the above analysis requires modification. On the other hand, groups separated by larger than the correlation length $\xi$ will be moving independently. Suppose two groups of length $\xi$ and having $\xi / d$ monopoles each are moving together, each at speed $v$. If $P_{x} \ll 1$, when the leading member of one group reaches the other, it will reflect. It will then move back toward its own group, reflect off the second member, and very soon the coherent motion 
will be destroyed. The monopoles will now be bouncing back and forth as above, and the analysis of Sec. [V] will apply.

On the other hand, if $P_{x} \approx 1$, the leading members of the groups will annihilate with each other, then the subsequent members in turn will do likewise. In time $\xi / v$ the trailing members of the two groups will reach each other and annihilate, and both groups will be gone from the string. Let us see if the existence of correlations changes our previous conclusions in this case.

We will consider the following simple model. Suppose that the string is split up into uncorrelated sections of length $\xi$ that are moving either to the right or to the left. This understates the annihilation rate, because in the real situation there are no precise trains moving in lockstep. Any given section will have the section ahead coming toward it on average half the time, in which case it will annihilate with that section in time $\tau=\xi / v$. Thus in time $\tau$, the number of monopoles will decrease by a factor of 2 , and so the average monopole distance $d$ will increase by that factor. We will approximate that in the same time, the motion of the string reorganizes the monopoles into sections with a new, larger value of $\xi$ and redistributes the monopoles and empty regions, so that we can once again treat the monopoles as uniformly distributed. The result is that $d$ doubles in time $\tau$.

Now, how large can $\xi$ be? From causality, the correlation length on the string cannot exceed vt. But it takes time for monopoles to forget their original velocities and acquire new ones from the moving string. So $\xi$ must be less than $v t$, and thus $\tau<t$. Consequently, when $t$ doubles, $d$ increases by more than a factor of 2 . Thus $r$ falls more quickly than $1 / t$.

This process begins when friction no longer prevents necklaces from achieving velocity $v$, at time $t_{\text {necklace }}$. According to the analysis above, $r$ would start to drop faster than as $1 / t$, which means that monopole density tracks the edge of the frictional regime, as described in Sec. IV, so $r \sim 1$ at $t=t_{*}$.

For light strings, this is sufficient to show that necklaces cannot be an observable source of cosmic rays, as follows. If $r \sim 1$ at $t=t_{*}$, then by Eq. (5),$\Omega_{M} \sim \mu / m_{\mathrm{pl}}^{2}$ then. After the initial symmetry breaking transition, there is no process that creates monopoles, so their number in a comoving region cannot change, and so $\Omega_{M}$ cannot increase from the time of matter-radiation equality, $t_{\text {eq }}$, until today. In fact, further annihilations will decrease $\Omega_{M}$, but we don't even need to consider that. In order to have $t_{*} \gtrsim t_{\mathrm{eq}} \sim m_{\mathrm{pl}} / T_{\mathrm{eq}}^{2}$ we require $\mu \lesssim m_{\mathrm{pl}} T_{\mathrm{eq}} \sim 10^{10} \mathrm{GeV}^{2}$, so $\Omega_{M} \lesssim T_{\mathrm{eq}} / m_{\mathrm{pl}} \sim 10^{-28}$, which is negligible. If string are lighter still, so that $t_{*}$ is in the future, then as long as we stay at the edge of the frictional regime, we have the unobservable case of Eq. (12).

If the string scale is larger, then $t_{*}$ will be in the radiation era. In that case, monopoles redshift like matter in the radiation era, so $\Omega_{M}$ grows proportionally to the scale factor, which in turn is proportional to $t^{1 / 2}$. Thus, again ignoring annihilations, we find that today

$$
\Omega_{M} \lesssim \frac{\mu}{m_{\mathrm{pl}}^{2}}\left(\frac{t_{\mathrm{eq}}}{t_{*}}\right)^{1 / 2} \sim \frac{\mu^{2} t_{\mathrm{eq}}^{1 / 2}}{m_{\mathrm{pl}}^{7 / 2}}
$$

To agree with Eq. (마) requires

$$
\mu^{2} \gtrsim 10^{-10} m_{\mathrm{pl}}^{7 / 2} t_{\mathrm{eq}}^{-1 / 2}
$$

or

$$
\mu \gtrsim 10^{19} \mathrm{GeV}^{2}
$$

If $\mu$ is too small to obey Eq. (17), then as long as the monopole density tracks the frictional regime until $t=t_{*}$, there will be no observable cosmic rays. 
For strings obeying Eq. (17), we note that after $t_{*}, r$ continues to drop as $1 / t$, so today we have

$$
r \sim \frac{t_{*}}{t_{0}}=\frac{m_{\mathrm{pl}}^{3}}{t_{0} \mu^{2}},
$$

and

$$
\Omega_{M} \sim \frac{m_{\mathrm{pl}}}{t_{0} \mu}<\sim 10^{-41}
$$

once again unobservably small.

One might also be concerned about the effect of statistical fluctuations on the number of monopoles moving in the two directions. Consider a long segment of string containing $N$ trains of monopoles. By chance we expect there to be of order $\sqrt{N}$ more trains moving in one direction than another. If the particle velocities were fixed over time, the left-movers and right-movers would annihilate with each other, leaving about $\sqrt{N}$ trains moving in the majority direction. However, in our case, the monopole motion is not fixed. Instead, as time passes, the motion of the string will affect the monopole motions in such a way that any time there will be roughly the same number going to two directions, rather than preserving any preexisting asymmetry. This prevents asymmetries from growing as a fraction of the total monopole density, and thus the effect described here is minimal.

\section{WIGGLY STRINGS}

A realistic string is not exactly straight. Instead, it has some spectrum of "wiggles". As monopole and antimonopole approach each other, the wiggles can be compressed between the two monopoles, similarly to photons between two mirrors, and contribute an effective repulsion. Could such a force prevent the monopoles from colliding? We will show that very large wiggles might be barely enough to prevent collisions, but that in fact one process or

another will always damp out the wiggles, so that a cosmologically significant number of monopoles will never remain.

\section{A. Force due to wiggles}

First let us analyze the repulsive effect of wiggles. We expect that after the monopole has moved in a given direction, it has a very wiggly string on one side and a much straighter string on the other side. The effective tension of the wiggly string could be as low as zero [2], while the effective tension on the straight side could be as high as $\mu$. One might think that the wiggles could exert a true repulsion that would add to the force on the straight string side of the monopole, but this is not correct. In a close-up picture of the monopole (e.g., Fig. 3), we see that the string moves around but always exerts an instantaneous force of $\mu$ in the direction in which it is pointing. If the set of directions of the string to the left of the monopole are uniformly distributed, this force could average to zero but even if this is not the case we would certainly not expect it to point in the direction opposite to the direction from one monopole to the other.

Now consider two monopoles moving toward one another. They will typically be separated by distance $d$. As we explained above the maximum value of the repulsive force is $\mu$ and can thus remove at most an amount of kinetic energy of the order of $\mu d=M / r$ as the monopoles come together. The typical velocity is $v$, so the total kinetic energy is $M v^{2} \sim M /(1+r)$. If 


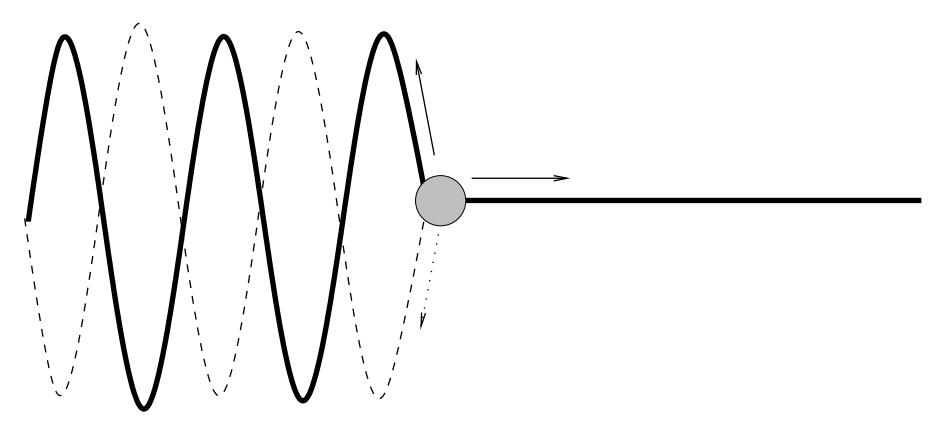

FIG. 3: Force on a monopole due to wiggles. The monopole is moving to the left, leaving a straight string to its right and a very wiggly string to its left. The straight string always exerts a force $\mu$ to the right. The wiggly string exerts an instantaneous force $\mu$ in a varying direction. For a sufficiently wiggly string the average of the varying force will be small, leaving a net rightward force $\mu$.

$r \gtrsim 1$, these are about equal, so the only most optimistic effective repulsion has the right size to bring the monopoles to a halt. On the other hand, if $r \ll 1$, then the repulsive force of wiggles can easily be sufficient. We will see in the following, however, that there are always processes that damp the wiggles, and so significant repulsive force is not available in situations of relevance to cosmic rays.

The same conclusions apply if the monopoles move in groups. Suppose there are two groups of length $\xi$, so each group contains $N=\xi / d$ monopoles and thus $N$ times more kinetic energy than in the single particle case. If the distance between group centers is also $\xi$, then the monopole kinetic energy that can be removed by the repulsive force of wiggles is also larger than the single particle case by factor $N$, so the comparison is just as before.

\section{B. Damping of wiggles}

Even if wiggles could in principle keep the monopoles from colliding, they would have to survive long enough to do so. Wiggles can be damped by friction or by gravitational back reaction, and we will see that one process or the other prevents there from being sufficient wiggliness to keep monopoles from colliding over a long period of the history of the universe.

First consider the frictional damping of wiggles on the string between a pair of monopoles. In the Appendix we see that wiggles on the string will be exponentially damped until the universe reaches the time $t_{*}$ when a string with a given $\mu$ would no longer be friction dominated. Thus wiggles do not affect string motion until $t_{*}$, and so, by the argument of Sec. V, only strings obeying Eq. (17) are of interest.

After $t_{*}$, frictional damping is ineffective, but now the strings are moving relativistically, and so they can be damped by the emission of gravity waves. Consider wiggles of wavelength $\lambda$ and amplitude $\epsilon \lambda$ on the string segment between two monopoles. The parameter $\epsilon$ is a dimensionless measure of the strength of the wiggle, and if $\epsilon \ll 0$, the wiggles increase the mass density and decrease the tension of the string by factor $1+\epsilon^{2} / 4$. Such wiggles emit gravity waves with power per unit length ${ }^{6}[19,20](\pi / 4) G \mu^{2} \epsilon^{4} / \lambda$. The energy emitted comes

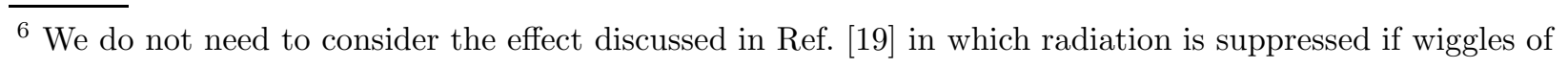


from the wiggles, so

$$
\frac{d}{d t} \epsilon^{2}=-\pi G \mu^{2} \epsilon^{4} / \lambda
$$

which has the solution

$$
\epsilon^{2}=\frac{\lambda}{\pi G \mu(t+\text { const })}
$$

If the wiggles had amplitude $\epsilon_{0}$ at some initial time, then an interval $\Delta t$ later the amplitude is

$$
\epsilon=\left(\frac{\lambda}{\pi G \mu \Delta t+\lambda / \epsilon_{0}^{2}}\right)^{1 / 2}
$$

Now repulsion can only result from wiggles whose wavelength obeys

$$
\lambda \mu \lesssim M
$$

Wiggles longer than this will travel past a monopole rather than being reflected, and so will not lead to a force on the monopole. Thus regardless of $\epsilon_{0}$, we find

$$
\epsilon \lesssim\left(\frac{M}{\pi G \mu^{2} \Delta t}\right)^{1 / 2}
$$

for relevant wiggles.

As argued above, we only need to consider $\mu \gtrsim 10^{19} \mathrm{GeV}^{2}$. With $\Delta t$ the present age of the universe, we find $\epsilon \lesssim 10^{-11}$, so primordial wiggles have been damped into insignificance by gravitational wave emission. In fact, after even the much shorter time $\Delta t=t_{*}$, we already have $\epsilon \lesssim \sqrt{M / m_{\mathrm{pl}}} \lesssim 10^{-1}$. By the argument of Sec. VIA such wiggles are too small to prevent monopoles from colliding if $r>1$.

Of course the wiggles might not be primordial. If wiggles are produced by intercommutations, then the timescale for production will be of order the Hubble time, since the distance between strings is of that order. Then the previous calculation applies and $\epsilon$ is trivial. Suppose instead that wiggles are produced by the oscillations of loops [17]. The smallest loop that can persist until the present day has size $l \sim G \mu t_{0}$. While such a loop might be producing wiggles as it oscillates, in one oscillation the wiggles will be damped to level $\epsilon \sim \sqrt{M /\left(G^{2} \mu^{3} t_{0}\right)} \sim 10^{-1}$. These wiggles will have no effect unless $r<1$. But in that case, $\Omega_{M}$ is too small to give observable effects unless $G \mu \gtrsim 10^{-10}$, in which case $\epsilon \lesssim 10^{-16}$.

Thus, repulsion due to wiggles can never revive necklaces as a source of observable cosmic rays, regardless of the string tension.

\section{MONOPOLES WITH UNCONFINED FLUXES}

If monopoles have an unconfined flux in addition to the flux carried by the strings, and there is a plasma of particles charged with respect to that flux, then a monopole will experience a frictional force [2] of order $T^{2} v$, which results in power dissipation of order $T^{2} v^{2}$. Comparing this with the monopole kinetic energy of order $M v^{2}$ yields a damping

different sizes are moving in the two directions. In order to exert a repulsion the wiggles must reflect off the monopoles and so move evenly in both directions. 


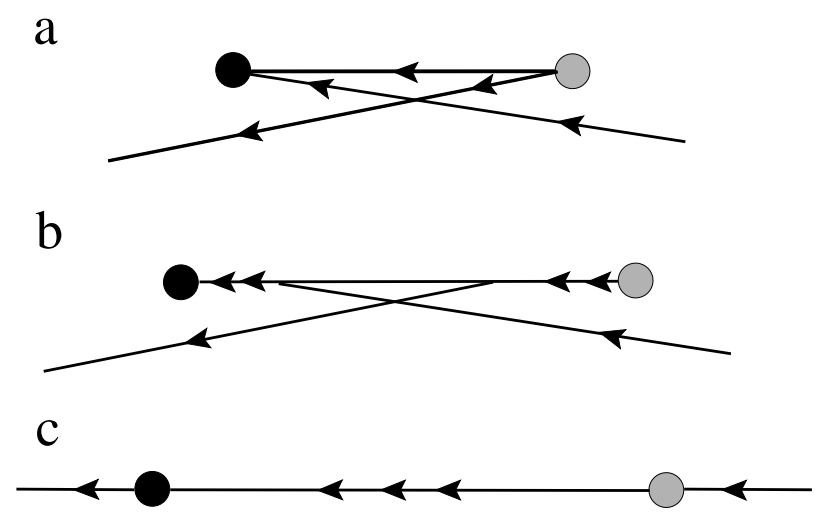

FIG. 4: If the monopoles are connected by Type I strings, regions of higher winding will easily be formed in the collisions between monopole and antimonopoles.

time $M / T^{2}$, which is always much less than the Hubble time, $m_{\mathrm{pl}} / T^{2}$. Thus as long as there is a background of charged particles, the monopole motion will be friction-dominated. In this case, the monopoles start to acquire significant velocities only when the charged particles annihilate, at the latest at the time of electron-positron annihilation, $t_{\mathrm{ee}}$.

In the standard scenario of Sec. IV], the monopoles begin annihilating at $t_{\mathrm{ee}}$, and the results of that section still apply. If instead the annihilation rate is reduced by the considerations of Sec. $\mathrm{V}$, the monopole density falls as $1 / t$. But even this is enough to prevent an observable signal. At the time of nucleosynthesis, which is essentially the same as $t_{\mathrm{ee}}$, we require $\Omega_{M} \lesssim 1$ to preserve the correspondence with observation of light element abundances. ${ }^{7}$ Between $t_{\mathrm{ee}}$ and $t_{\mathrm{eq}}$, annihilations decrease the number of monopoles in a comoving volume by factor $t_{\mathrm{eq}} / t_{\mathrm{ee}} \sim\left(T_{\mathrm{ee}} / T_{\mathrm{eq}}\right)^{2}$. Meanwhile, redshifting of relativistic particles decreases the energy in a comoving volume by factor $T_{\text {ee }} / T_{\text {eq }}$, leading to a net decrease in $\Omega_{M}$ by factor $T_{\text {ee }} / T_{\text {eq }} \sim 10^{6}$. After equality, $\Omega_{M}$ simply declines as $1 / t$, producing an additional decline of $t_{0} / t_{\text {eq }} \sim 10^{6}$. Thus today we have $\Omega_{M} \lesssim 10^{-12}$ in disagreement with Eq. (6) .

\section{TYPE I STRINGS}

One can also imagine that the strings that connect the monopoles are Type I strings. These are characterized by an attractive force between parallel static strings [23]. In this case the energy density of an $n=3$ string is less than the sum of an $n=2$ string and $n=1$ string. This makes the detachment process shown in Fig. 1 energetically disfavored. Furthermore, if the strings form a "zigzag" string as in Fig. 2, it is possible for them to form a "zipper" region where two unit-winding strings become attached to one another [24]. Thus instead of the result shown in Fig. 2, we have that shown in Fig. 4, in which the particles, after initially passing outside the string nevertheless end up in a single string.

If the string is sufficiently Type I, perhaps all detachment processes are suppressed, so that $P_{d}=0$. Instead of detachment, we end up with the monopole and antimonopole moving

\footnotetext{
7 This is simply the constraint that the universe should be radiation dominated during nucleosynthesis. A much stronger bound can be derived by considering effects of energetic particles released by monopole annihilation during nucleosynthesis.
} 
away from each other inside a single string. The tension of the $n=3$ string in between the particles will eventually reverse their motion and bring them back past each other. In this case, there will be a probability for annihilation on the return trip. If this probability is large enough, the monopole density will still be greatly decreased.

We can estimate the probability of annihilation as the geometrical ratio between the size of the monopole to the string thickness,

$$
P_{x}=P_{a}=\left(\frac{\eta_{s}}{\eta_{M}}\right)^{2}=\frac{\mu}{M^{2}}
$$

In anything but the most extreme cases, this gives $P_{x}>10^{-28}$, and thus by the argument of Sec. IV. $\Omega_{M}$ is negligible. If we go to $M=10^{17} \mathrm{GeV}, \mu=(100 \mathrm{GeV})^{2}$, with no probability for detachment, Eq. (25) gives $P_{x} \sim 10^{-30}$. In such a case, Eq. (13) and Eq. (5) give

$$
\begin{aligned}
r & \sim\left(\frac{M}{\mu t_{0} P_{x}}\right)^{2} \\
\Omega_{M} & <\frac{M^{4}}{\mu^{3} t_{0}^{4} P_{x}^{4} m_{\mathrm{pl}}^{2}}=\frac{M^{12}}{\mu^{7} t_{0}^{4} m_{\mathrm{pl}}^{2}}<10^{-26} .
\end{aligned}
$$

\section{SUPERCONDUCTING STRINGS}

The strings formed in the second phase transition may have some massless zero modes living on them, in other words they may be superconducting ${ }^{8}$ [21]. These new degrees of freedom change the effective equation of state of the string and could have potentially important consequences for the motion of the string itself [2]. Here we will be mostly interested on the effect that these modes may have on the longitudinal velocity of the monopoles.

We can think of a superconducting string as a wire along which massless charge carriers can flow. ${ }^{9}$ These charge carriers act as a one-dimensional gas and exert pressure that decreases the tension of the string. If the charge carriers can pass freely across a monopole, then they have no effect on monopole motion along the string. ${ }^{10}$ But if the charge carriers are reflected by the monopole, they can be concentrated between the monopole and antimonopole as they approach. Reflection reverses the current but retains the charge of the carrier, so the string between monopoles will have charge but no current. The charge density cannot grow without bound: eventually the charge carriers can scatter and leave the string. The maximum charge density is dependent on the specific model. If charge carriers can scatter to produce light charged particles not bound to the string, the maximum charge density will be low. If not, it can be high enough to substantially reduce the string tension,

\footnotetext{
${ }^{8}$ Note that we use the terminology of superconducting to refer to any kind of persistent currents created by the zero modes localized on the string core and not only to the electromagnetically charged modes.

${ }^{9}$ Here we are thinking of fermionic superconducting zero modes, but bosonic superconductivity leads to similar limits.

${ }^{10}$ Charge carriers are held to the string because they are massless inside but massive outside the string. The existence of monopoles puts only a small gap in the string core, and it seems that charge carriers could easily tunnel across this gap.
} 
but it can never reduce it to zero [22]. As discussed in Sec. VIA, this means that the effective repulsion is too small to prevent collisions if $r>1$, but might do so if $r<1$. Thus there may be a small window for annihilations to stop if the string is superconducting, the scale of superconductivity is nearly the same as the string scale, and the string acquires a significant charge density. This process works only if $r<1$, so $\Omega_{M} \lesssim G \mu$, and thus it is only of interest for heavy strings with $G \mu \gtrsim 10^{-10}$.

\section{DISCUSSION}

We have shown that the monopoles on a necklace acquire typical velocities along the string large enough to lead to very frequent interactions between monopoles and antimonopoles. We have studied a wide variety of cases (strings of Type I or II, superconducting strings, strings with wiggles, monopoles with additional unconfined fluxes), but found in almost every case that interactions lead, at early times, to monopole-antimonopole annihilation or to the monopoles leaving the string in monopole-antimonopole pairs. In the former case the necklaces just become ordinary cosmic strings. In the latter case, instead of necklaces we have a population of monopole-antimonopole pairs, and the subsequent evolution is just as we discussed in Ref. [8]. In neither case does the possibility of necklaces add anything to the set of possible ultra-high-energy cosmic ray sources.

The exception to the above is heavy superconducting strings with heavy condensate scale. In this case it seems barely possible that the superconducting charge carriers could prevent the monopoles from reaching each other. In order to make a viable model, the string scale must be quite high, $G \mu \gtrsim 10^{-10}$, the scale of the condensate must also be quite high, and there must not be any process which scatters condensate particles into lighter particles that are not bound to the string. Furthermore, the charge carriers must not be able to pass or tunnel between the segments of string on the two sides of a monopole, and there must be some mechanism by which the string acquires a significant fraction of the maximum charge density.

\section{Acknowledgments}

We thank Marco Kneipp, Xavier Siemens, Alex Vilenkin and Tony Weinbeck for helpful conversations. K.D.O. was supported in part by the National Science Foundation under grants 0457456 and 0855447.

\section{Appendix A: Friction}

In this appendix, we compute the time at which friction is important for segments of string between monopoles and the time at which friction is important for the motion of the necklace as a whole. If $r$ is significant, friction loses its effect sooner for the necklace as a whole, because its velocity in the absence of friction is lower.

The frictional force per unit length on a string with velocity $v_{s}$ interacting with a thermal background of temperature $T$ goes as [18]

$$
F \sim T^{3} v_{s}
$$


so the damping power per unit length is

$$
F v_{s} \sim T^{3} v_{s}^{2}
$$

We neglect relativistic factors here. String segments between monopoles are never ultrarelativistic, so the contribution of relativistic effects is at most of order 1 .

Excitations of wavelength $\lambda$ and amplitude $A$ have energy per unit length of order $\mu A^{2} / \lambda^{2}$. They give rise to a string velocity of order $A / \lambda$, so the frictional power per unit length is $T^{3} A^{2} / \lambda^{2}$ and the damping timescale is [2].

$$
\tau_{d} \sim \frac{\mu}{T^{3}} .
$$

Features on the string will be exponentially damped, as long as $\tau_{d}$ is less than the age of the universe. In the radiation era, that is $t \sim m_{\mathrm{pl}} / T^{2}$, so we find that friction domination for string segments ends at

$$
t_{*} \sim \frac{m_{\mathrm{pl}}^{3}}{\mu^{2}} \quad \text { (Radiation) } .
$$

Although we did the calculation in a slightly different way, this is just time when friction stops being important for a string network without monopoles [2].

If friction lasts until the matter era, we have instead $t \sim m_{\mathrm{pl}} / \sqrt{T^{3} T_{\mathrm{eq}}}$, so

$$
t_{*} \sim \frac{m_{\mathrm{pl}}^{2}}{\mu T_{\mathrm{eq}}} \quad \text { (Matter) }
$$

Now let us consider the damping of the necklace as a whole. The overall motion has $v \sim 1 / \sqrt{1+r}\left[\underline{6}\right.$, kinetic energy per unit length of order $(1+r) \mu v^{2} \sim \mu$, and frictional power per unit length of order $T^{3} /(1+r)$. The damping time for this case is thus

$$
\tau_{d} \sim \frac{(1+r) \mu}{T^{3}}
$$

and friction for the motion of the necklace as a whole ends at time $t_{\text {necklace }}$, where

$$
\begin{aligned}
& t_{\text {necklace }} \sim \frac{m_{\mathrm{pl}}^{3}}{(1+r)^{2} \mu^{2}} \quad(\text { Radiation }) \\
& \left.t_{\text {necklace }} \sim \frac{m_{\mathrm{pl}}^{2}}{(1+r) \mu T_{\mathrm{eq}}} \quad \text { (Matter }\right) .
\end{aligned}
$$

[1] D. J. Bird et al. [HIRES Collaboration], Astrophys. J. 424, 491 (1994).

[2] A. Vilenkin and E.P.S. Shellard, "Cosmic Strings and Other Topological Defects", Cambridge University Press, 1994.

[3] M. B. Hindmarsh and T. W. B. Kibble, "Cosmic strings," Rept. Prog. Phys. 58, 477 (1995).

[4] P. Bhattacharjee and G. Sigl, Phys. Rept. 327, 109 (2000).

[5] V. Berezinsky, P. Blasi and A. Vilenkin, Phys. Rev. D 58, 103515 (1998).

[6] V. Berezinsky and A. Vilenkin, Phys. Rev. Lett. 79, 5202 (1997). 
[7] V. K. Dubrovich and M. Y. Khlopov, JETP Lett. 77, 335 (2003) [Pisma Zh. Eksp. Teor. Fiz. $\mathbf{7 7}, 403(2003)]$

[8] J. J. Blanco-Pillado and K. D. Olum, Phys. Rev. D 60, 083001 (1999).

[9] M. Hindmarsh and T. W. B. Kibble, Phys. Rev. Lett. 55 (1985) 2398.

[10] M. Aryal and A. E. Everett, Phys. Rev. D 35, 3105 (1987).

[11] T. Matsuda, JHEP 0505, 015 (2005). T. Matsuda, JHEP 0604, 017 (2006). T. Matsuda, JCAP 0604, 005 (2006).

[12] K. Dasgupta, H. Firouzjahi and R. Gwyn, JHEP 0704, 093 (2007).

[13] L. Leblond and M. Wyman, Phys. Rev. D 75, 123522 (2007).

[14] K. Dasgupta, H. Firouzjahi and R. Gwyn, JHEP 0704, 093 (2007).

[15] L. Leblond, B. Shlaer and X. Siemens, Phys. Rev. D 79, 123519 (2009).

[16] M. Lake, S. Thomas and J. Ward, arXiv:0906.3695 [hep-ph].

[17] X. Siemens, X. Martin and K. D. Olum, Nucl. Phys. B 595 (2001) 402.

[18] A. E. Everett, Phys. Rev. D 24, 858 (1981).

[19] X. Siemens and K. D. Olum, Nucl. Phys. B 611, 125 (2001) [Erratum-ibid. B 645, 367 (2002)]

[20] M. Hindmarsh, Phys. Lett. B 251, 28 (1990)

[21] E. Witten, Nucl. Phys. B 249, 557 (1985).

[22] P. Peter, Phys. Rev. D 45, 1091 (1992).

[23] L. Jacobs and C. Rebbi, Phys. Rev. B 19, 4486 (1979).

[24] L. M. A. Bettencourt, P. Laguna and R. A. Matzner, Phys. Rev. Lett. 78, 2066 (1997). 